\title{
Experimentieren um einen Satz zu finden - vollständing separierbare Mosaike auf der Kugel und ihre Anwendungen
}

\author{
ÉVA VÁSÁRHELYI
}

\begin{abstract}
This paper reports a case-study which took place within the project named „Inner differentiation and individualization by creating prototypes and analogies under consideration of motivational constraints (taking into account computer-based teaching and learning) " as a part of a pre-service teacher training at the University of Salzburg (Herber, H.-J. \& Vásárhelyi, É.).

The goal of the experiment was to help students to learn the fundamental concepts and basic constructions of spherical geometry using the Lénárt Sphere (a transparent plastic ball with construction-tools) and some self-made interactive worksheets with the Windows version of the dynamical geometry software Cabri.
\end{abstract}

Key words and phrases: Hochschuldidaktik, Computereinsatz, Diskrete Geometrie.

ZDM Subject Classification: G60, G90, U50, U60.

In diesem Artikel wird über eine Falluntersuchung berichtet, die im Rahmen eines Forschungsprojektes (Innere Differenzierung, Analogiebildung und Computereinsatz, Herber, H.-J. \& Vásárhelyi, É.) durchgeführt wurde.

Versuchspersonen: Lehramtskandidaten des zweiten Studienabschnittes im Rahmen der Lehrveranstaltung „Spezielle Themen aus der Didaktik der Mathematik - Trigonometrie". ${ }^{1}$

${ }^{1}$ Die Studierenden haben in dem ersten Studienabschnitt im Rahmen der mathematischen Grundausbildung Geometrie gelernt. Die Endphase der Untersuchung, in der die Aufgabe sehr komplex geworden ist, haben zwei Studierenden (von fünf) mitgemacht. 
Ziele und Methode: Die Lehrveranstaltung hat eine didaktische Zusammenfassung zum Thema Trigonometrie angezielt: Geschichte der Trigonometrie, Winkel im Bogenmaß, das rechtwinklige Dreieck, Übergang zum allgemeinen Dreieck, die Graphen der Winkelfunktionen, zusammengesetzte Funktionen (Schwingungen), die allgemeine Sinusfunktion, Rechnen mit Winkelfunktionen, Additionstheoreme, Ableitungen der trigonometrischen Funktionen, Anwendungen, sphärische Trigonometrie (Kugel). Während der Lehrveranstaltung haben die Studierenden traditionelle (Konstruktionswerkzeuge, Modelle, Arbeitsblätter, ... ) sowie elektronische (Animationen, interaktive Arbeitsblätter, E-Buch, ... ) Unterlagen bzw. Anschauungsmittel bekommen, die von ihnen bearbeitet, ergänzt bzw. korrigiert wurden. Diese Kombination der Lernmaterialien wurde unter der von mehreren Falluntersuchungen ${ }^{2}$ bestätigten Annahme gewählt, dass sie

- die Analogiebildung (im direkten „händischen“ Umgang Gelerntes auf computerbasierte Prozesse transferieren, Abstrahieren von den unterschiedlichen enaktiven Prozessen zu den ikonischen, semantischen und auch enaktiven Tiefenrepräsentationen) unterstützen und bewusst machen kann,

- das Protokollieren des Unterrichtsgeschehens für die Studierenden und für die Lehrperson erleichtert, und

- das kreative Umgehen mit dem Lehrstoff durch die freie Wahl von Mitteln und Annäherungsweisen ermöglicht.

In der Endphase der Lehrveranstaltung (Anwendungen der Trigonometrie) wurde ein Aufsatz ${ }^{3}$ aus dem Gebiet der diskreten Geometrie (Vásárhelyi 1991, 2003a) in 4 Doppelstunden durchgearbeitet - mit den folgenden spezifischen Lehr-/Lernzielen:

- ein Beispiel zeigen, wie die Trigonometrie in der aktuellen Forschung anzuwenden ist und dabei das Lesen, Verstehen und Interpretieren eines wissenschaftlichen Artikels auf diesem Gebiet geübt werden kann;

- die Zusammenwirkung der unterschiedlichen Repräsentationen (Modelle, Skizzen, Konstruktionen, Animationen, synthetische und analytische Beschreibungen, ... ) betonen und dadurch einige fundamentale Konzepte und Grundkonstruktionen der sphärischen Geometrie und deren Verbindungen mit der Euklidischen Geometrie entdecken lassen.

${ }^{2}$ Fuchs \& Vásárhelyi 1998, Vásárhelyi 1999, Herber \& Vásárhelyi 2003.

${ }^{3}$ Auch der mathematische Teil des hier vorgelegten Aufsatzes ist eine Erstveröffentlichung. Eine ungarische Version ist im Internet unter www.mathdid.inhun.com erreichbar. 
Alle Studierenden haben den mathematisch korrekt geschrieben Text Schritt für Schritt (als I. Problembegegnung, II. Begriffsexplikation, III. Transferphase/Experimentieren, IV. Problemlösen) ohne Abbildungen bekommen. Als Anschauungsmittel wurden Cabri-Arbeitsblätter (Vásárhelyi 2003b), die Lénárt Kugel (Lénárt, I., Parisot, K. J., Vásárhelyi, É. 2002) und andere Medien nach freier Wahl verwendet.

Um mit der dreidimensionalen Konfiguration besser umgehen zu können, wurde das sphärische Konstruktionswerkzeug, die Lénárt-Kugel, eingesetzt. Man kann unmittelbar auf die Plexiglaskugel zeichnen oder halbkugelförmige Folien als sphärisches Zeichenbrett verwenden (Abb. 1). Um die Erfahrungen zeichnerisch protokollieren zu können, wurden die halbkugelförmigen Folien mit Hilfe des Overheadprojektors auf die Wand projiziert.

Die Computeranimationen ermöglichen weitere Verbindungen zwischen dem konkret-manipulativen Experimentieren und der zweidimensionalen Darstellung: Die interaktiven Arbeitsblätter enthalten dynamische Konstruktionen, die mittels individueller direkter Manipulation („Drehen“ und „Kippen“) oder durch automatisierte Bewegung („Animation“) jede Projektionsrichtung einstellen, sogar „kontinuierlich“ variieren lassen. Durch die händische Einstellung kann die für das Zeichnen, Rechnen, Drucken, ... günstige Projektion ausgesucht werden (Abb. 2a). Durch die Folge der zweidimensionalen Parallelprojektionen entsteht individuelle Erfahrung mit beweglichen Figuren in dem Sinne, dass sich die Dimensionierung als Ganzes verändert: die Folge der Bildschirmfiguren wird vom Beobachter als dreidimensionales Gebilde wahrgenommen (Abb. 2b). (Dies entspricht dem bekannten gestaltpsychologischen Phi-Phänomen von Wertheimer 1912.)

Die Arbeitsblätter sind so gestaltet, dass der Benützer auf die Kugel zeichnen und jeder Zeit wählen kann, ob er keine Hilfe, wenig Hilfe oder eine vollständige Lösung und Erklärung in Anspruch nimmt (Abb. 3).

\section{Didaktische Begründung der Themenwahl und der Vorgehensweise:}

In unserer natürlichen und künstlichen Umgebung kommt die Kugel oft vor: ein Ball, Früchte, Seifenblasen, Viren, der Augapfel, die Gelenke, die elementaren Körperchen, die Raumkörper stehen der Kugel viel näher als der Ebene. Die Kugel wird in wissenschaftlichen Modellen und technischen Werkzeugen oft benutzt. Unseren Globus kann man mit guter Annäherung als Kugel betrachten (Geographie, Kartographie, Erdgeschichte, Meteorologie, Navigation, Chemieindustrie, Biologie, Umweltschutz, Geometrie, Technisches Zeichnen, Darstellende Geometrie, Informatik, Elektronik, Architektur, Kunst, Design, Industrie- und 
Maschinenbau, Verpackungstechnik, ...). Das Kugelmodell spielt eine zentrale Rolle in zahlreichen Bereichen der klassischen Physik (Kinematik, Thermodynamik, Elektrizitätslehre, ... ).

Mit Absicht wurde ein (auch für die Studierenden) komplexes Thema gewählt, damit diverse Querverbindungen und Analogiebildungen - mit entsprechender ästhetischer Konnotation - angesprochen werden können: Gestalt und geometrischer Begriff; synthetische und analytische Charakterisierung von Objekten und Relationen (Geometrie und Algebra); euklidische Geometrie und Geometrie auf der Kugeloberfläche; dreidimensionale Konfigurationen und ihre zweidimensionalen Darstellungen (Skizze, Projektion durch Zentralperspektive und Parallelprojektion).

Auf Grund unterschiedlicher Begabungen und Interessen sind jedoch bei den Studierenden individuelle Unterschiede beim Verstehen eines Aufsatzes, Beweises (konkret, Beweisidee, Sinn des Satzes, Anwendungsbereich) zu erwarten. Während der Lehrveranstaltung wurde den Studierenden ein differenziertes Angebot von Erarbeitungsaufgaben unterschiedlichen Anspruchsniveaus angeboten.

Erfahrung: Es hat sich herausgestellt, dass die Abbildungen auch dann nötig sind, wenn der Text mathematisch korrekt geschrieben ist. In eckigen Klammern befinden sich die Hinweise auf die Figuren, die von den Studierenden zum Verständnis benötigt wurden, bzw. ist protokollarisch angegeben, wo und welche Anschauungsmittel die Studierenden verwendet haben.

Obwohl hier studentische Reaktionen aufgezeigt werden, wird nicht auf die Denkstrukturen von Studierenden eingegangen, weil das Anliegen des Aufsatzes in der Planung eines Hochschulunterrichts des mathematischen Inhaltes im Vordergrund gestanden ist. Die studentischen Reaktionen werden aufgenommen um die Notwendigkeit aufzuzeigen, den mathematischen Inhalt sowohl in sprachlichsymbolischer als auch in grafischer Darstellung den Studierenden anzubieten, wenn Verständnis für den mathematisch-geometrischen Inhalt erwartet wird.

Didaktische Schlussfolgerung: Die symbolische Repräsentation (auch in voll verbalisierter Form) garantiert in der Erstbegegnung nicht ein hinreichendes (analytisch-synthetisches) Verfügen über das Stoffgebiet (auch Studierende der Mathematik brauchen bei einem komplexen, dreidimensionalen Problem enaktive und ikonische Stützung).

Im folgenden Bericht werden die Abbildungen am Ende gesondert dargestellt, damit der Leser die der Lernsituation ähnliche Wahl hat, den Text mit oder ohne Figuren zu lesen. In der didaktischen Originalsituation waren die Abbildungen 
am Bildschirm farbig und dynamisch, während die Abbildungen hier schwarzweiß und statisch sind.

PHASE I: Problembegegnung, Geschichte des Problems, Anwendungen

A) Mathematischer Inhalt

Die dichtesten Packungen bzw. dünnsten Überdeckungen von kongruenten Kreisen auf der Kugel ${ }^{4}$ sind nicht nur für die Mathematik interessant, sondern auch wegen ihrer Anwendungsmöglichkeiten in der Biologie (Tammes 1930, Goldberg 1967, Tarnai 1984), Chemie (Melnyk \& Knop \& Smith 1977) und Informationstheorie (van der Waerden 1961). Die systematische Untersuchung innerhalb der diskreten Geometrie stammt von L. Fejes Tóth. Er hat Dichteabschätzungen bezüglich Packungen und Überdeckungen angegeben, die für die Kreisanzahl $n=3,4,6,12$ die bestmöglichen Werte sind. Die dichtesten Packungen wurden für $n=5,7,8,9$ von Schütte \& van der Waerden (1951), für $n=10$ von Danzer (1975) und von Hárs (1986), für $n=11$ von Danzer (1975) und von Böröczky (1983), für $n=24$ von Robinson (1961) bestimmt. Die dünnsten Überdeckungen wurden für $n=5,7$ von Schütte (1955) und für $n=10,14$ von G. Fejes Tóth (1969) bestimmt.

\section{B) Medieneinsatz, Studentenaktivität}

- Internetsuche thematisch und nach Namen (einige Beispiele aus dem Suchergebnis in April 2003):

KUGELGEOMETRIE: www.didaktik.mathematik.uni-wuerzburg.de

www.home.unix-ag.org/scholl/kugelgeometrie

www.lehrer.uni-karlsruhe.de/ za382/mag/mag9798

rueckert-gym.de/facharbeiten/P06b.html

TAMMES + POLLEN: Optimal arrangements of $\mathrm{n}$ points on a sphere and in a circle

lp.fmf.uni-lj.si/plestenjak/talks/preddvor.pdf

Logarithmic points on the Sphere users.ipfw.edu/dragnevp/Sigma_Xi1.ppt

${ }^{4}$ Eine dichteste Packung bzw. dünnste Überdeckung zu suchen, bedeutet bei gegebener Anzahl der Kreise einerseits den größtmöglichen bzw. kleinstmöglichen Kreisradius zu bestimmen, andererseits die Lage der Kreise bei der optimalen Anordnung zu beschreiben. 
GOLDBERG: mathstat.uoguelph.ca/faculty/smith/full_pubs.pdf

MELNYK: mathworld.wolfram.com/SpherePacking.html

FEJES: eddy.uni-duisburg.de/treitz/denkmnu/fejes/fejes2.htm

- Experimentieren mit der Lénárt-Kugel und mit der Cabri-Darstellung der Kugel: Als Wiederholung und unmittelbare Anwendung des eben behandelten Lehrstoffes (Winkelfunktionen, Trigonometrie, Vektorgeometrie, geographische bzw. sphärische Koordinaten, orthogonale Axonometrie) wurde der mathematische Hintergrund der Darstellung (Steuerungs- und Konstruktionselemente, wie Drehen und Kippen, Punkt, Längen- und Breitenkreise konstruieren) diskutiert (Abb. 4, 5, siehe auch die Animationsliste).

\section{PHASE II: Begriffsexplikation}

\section{A) Mathematischer Inhalt}

\section{Spezielle Packungen - Separierbarkeit}

Definition 1 (L. Fejes Tóth \& G. Fejes Tóth 1973): Eine Packung ist vollständig separierbar, wenn sich jedes Paar durch eine solche Gerade trennen lässt, die keinen inneren Punkt der weiteren Elemente enthält [Abb. 6].

\section{Erweiterung der Definition}

Definition 2 (G. Fejes Tóth 1987): Ein System $\{S\}$ ist vollständig separierbar, wenn ein solches System $\left\{S^{\prime}\right\}$ existiert ${ }^{5}$, das eine vollständig separierbare Packung bildet und jedes Element von $\{S\}$ ein Element von $\left\{S^{\prime}\right\}$ enthält, so dass die Vereinigungsmenge von $\{S\}$ mit der Vereinigungsmenge von $\left\{S^{\prime}\right\}$ übereinstimmt [Abb. 7].

\section{Analoge Definition bezüglich der Kugelfläche}

Spielt die Rolle der Geraden der Großkreis, sind obige Definitionen auf die Kugel übertragbar. K. Böröczky hat die vollständig separierbaren Packungen von kongruenten Kreisen auf der Kugel für $n \leq 12$ bestimmt und er hat eine Konstruktion für $n=14$ gegeben.

Wir werden die dünnste Überdeckung der Kugel durch $n \leq 16$ vollständig separierbare kongruente Kreise bestimmen.

${ }^{5}$ Der innere Teil von $S^{\prime}$ darf dabei nicht leer sein. 


\section{B) Medieneinsatz, Studentenaktivität}

Experimentieren mit der Lénárt-Kugel und mit der Cabri-Darstellung der Kugel: Kreis, Gerade, Winkel zeichnen, Länge und Winkel messen, Elemente der sphärischen Trigonometrie erarbeiten (Abb. 8).

\section{PHASE III: Transferphase/Experimentieren}

\section{A) Mathematischer Inhalt}

\section{Vollständig separierbare Mosaike auf der Kugel}

Bildet ein System von Figuren eine vollständig separierbare Überdeckung auf der Kugel, dann existiert nach der Definition 2 ein Mosaik auf der Kugel, das durch endlich viele Großkreise definiert ist und dessen Zellen in je einem Element des Systems enthalten sind.

Der Kugelmittelpunkt ist das gemeinsame Symmetriezentrum der Großkreise und der Kugel. Daraus folgt, dass die Anzahl der Kreise bei einer vollständig separierbaren Überdeckung eine gerade Zahl ist. Der Grad der Eckpunkte eines vollständig separierbaren Mosaiks ist eine gerade Zahl und mindestens 4. Mit Hilfe dieser Eigenschaft und des Eulerschen Polyedersatzes lässt sich leicht kontrollieren, ob die Tabelle für $n \leq 16$ alle topologischen Möglichkeiten des vollständig separierbaren Mosaiks enthält ( $n$ ist die Anzahl der Zellen, $k$ ist die Anzahl der separierenden Großkreise, $e$ ist die Anzahl der Eckpunkte und $e_{i}$ ist die Anzahl der Eckpunkte vom Grad $i$ ). Bei einem Mosaik vom Typ A schneiden die separierenden Großkreise einander in demselben diametral gegenüberliegenden Punktpaar. Schneiden $k-1$ separierende Großkreise einander in demselben diametral gegenüberliegenden Punktpaar und werden alle durch einen weiteren (das Punktpaar nicht enthaltenen) Großkreis geschnitten, ist der Mosaik vom Typ B. Bei einem Mosaik vom Typ C schneiden die separierenden Großkreise einander paarweise in unterschiedlichem Punktpaar. 


\begin{tabular}{|c|c|c|c|}
\hline $\begin{array}{c}\text { Anzahl der } \\
\text { Zellen }\end{array}$ & $\begin{array}{c}\text { Typ des } \\
\text { Mosaiks }\end{array}$ & $\begin{array}{c}\text { Anzahl der } \\
\text { separierenden Großkreise }\end{array}$ & $\begin{array}{c}\text { Anzahl der } \\
\text { Eckpunkte }\end{array}$ \\
\hline$n=2$ & eindeutig & $k=1$ & $e=e_{4}=2$ \\
\hline$n=4$ & eindeutig & $k=2$ & $e=e_{6}=2$ \\
$e=e_{8}=2$
\end{tabular}

B) Medieneinsatz, Studentenaktivität

Experimentieren mit der Lénárt-Kugel und mit der Cabri-Darstellung der Kugel, die Erfahrungen skizzieren, einordnen (Abb. 9).

PHASE IV: Problemlösen, einen Satz finden

A) Mathematischer Inhalt

\section{Obere Abschätzung durch Konstruktion}

Um die dünnste Überdeckung der Kugel durch $n \leq 16$ vollständig separierbare kongruente Kreise zu finden, suchen wir die vollständig separierbaren Mosaike, deren „größte" Zelle durch den kleinstmöglichen Kreis überdeckbar ist (MinimaxPrinzip). Als Ausgangspunkt betrachten wir die „regelmäßigen“ Anordnungen der separierenden Kreise und bestimmen für diese den Überdeckungsradius. Dadurch erhalten wir eine obere Abschätzung für die allgemeinen Anordnungen [Abb. 10]. Für $\boldsymbol{n}=\mathbf{2}, \mathbf{4}, \mathbf{6}, 10$ genügt es zu bemerken, dass die Kreise vom Radius

$$
r_{2}=r_{4}=r_{6}=r_{10}=90^{\circ}
$$

um die Eckpunkte eines in einem Großkreis beschriebenen regelmäßigen $2 i$-Ecks $(i=1,2, \ldots)$ eine vollständig separierbare Überdeckung bilden. 
Für $\boldsymbol{n}=\mathbf{8}$ bilden die Flächenumkreise des regulären Mosaiks $\{3 ; 4\}$ eine vollständig separierbare Überdeckung ${ }^{6}$. Der Umkreisradius ist

$$
r_{8}=\operatorname{arcctg} \frac{\sqrt{2}}{2} .
$$

Für $\boldsymbol{n}=\mathbf{1 2}$ betrachten wir das Mosaik, das aus Kugeldreiecken mit den Winkeln $60^{\circ}, 90^{\circ}, 90^{\circ}$ besteht. Der Umkreisradius eines solchen Dreiecks ist

$$
r_{12}=\operatorname{arcctg} \frac{\sqrt{3}}{2}
$$

$\boldsymbol{n}=$ 14: Der Umkreisradius der Vierecksflächen des halbregulären Mosaiks ${ }^{7}$ $(4,3,4,3)$ ist

$$
r_{14}=45^{\circ}
$$

und das ist größer als der Umkreisradius der Dreiecksflächen. Das vollständig separierbare Kreissystem entsteht dadurch, dass die Umkreise der Dreiecksflächen durch die größeren Kreise ersetzt werden.

Der Umkreisradius eines Kugeldreiecks mit den Winkeln $45^{\circ}, 90^{\circ}, 90^{\circ}$ ist

$$
r_{16}=\operatorname{arcctg} \sqrt{\frac{\sqrt{2}+2}{4}},
$$

und aus diesen Kugeldreiecken kann ein Mosaik vom Typ B für $\boldsymbol{n}=\mathbf{1 6}$ gebaut werden.

Damit wurde für $n \leq 16$ durch Konstruktion gezeigt, dass eine vollständig separierbare Überdeckung durch kongruente Kreise existiert, bei denen der Radius $r_{n}$ ist, folglich ist der Radius bei der dünnsten Überdeckung höchstens $r_{n}$.

\section{Vermutung}

Die Regelmäßigkeit der Mosaike stellt eine Extremwerteigenschaft dar, bei einer vollständig separierbaren Überdeckung für $n \leq 16$ ist der Radius der kongruenten Kreise mindestens $r_{n}$.

\footnotetext{
${ }^{6}$ Das Symbol $\{p ; q\}$ bedeutet ein regelmäßiges Mosaik, in dem sich um jeden Eckpunkt $q$ regelmäßige $p$-Ecke anschließen. $\{3 ; 4\}$ ist das Analogon des regelmäßigen Oktaeders.

${ }^{7}$ Bei einem archimedischen halbregulären Mosaik sind die Flächen regelmäßig, und um jeden Eckpunkt schließen sich die in der Klammer aufgezählten regelmäßigen Vielecke an.
} 


\section{Beweis der Vermutung}

\section{Die separierenden Großkreise schneiden einander}

\section{in demselben Punktpaar}

$\boldsymbol{n}=\mathbf{2}$ : Überdecken zwei Kreise die Kugel, dann enthält - wegen der Separierbarkeit - jeder Kreis mindestens eine Halbkugel, deswegen ist der Radius mindestens $90^{\circ}$. Im Falle vom $90^{\circ}$ handelt es sich um diametral gegenüberliegende Kreismittelpunkte.

$\boldsymbol{n}=4,6,10$ : Der Aufbau des vollständig separierbaren $n$-flächigen Mosaiks ist topologisch eindeutig: alle separierenden Großkreise schneiden einander in demselben diametral gegenüberliegenden Punktpaar, z.B. in $\left(P, P^{\prime}\right)$. Der Durchmesser der Fläche ist $180^{\circ}$. Das bedeutet für den Radius der überdeckenden Kreise $r \geq 90^{\circ}$. Im Falle vom $90^{\circ}$ liegen die Kreismittelpunkte auf einem Großkreis mit den Polen $P$ und $P^{\prime}$. (Dies erlaubt dennoch unendlich viele Anordnungen.)

Für $\boldsymbol{n}=\mathbf{8}, \mathbf{1 2}, \mathbf{1 4}, 16$ lassen sich zwei vom Typ unterschiedliche, vollständig separierbare $n$-flächige Mosaike generieren. Beim Typ A gilt $r \geq 90^{\circ}$ für den Radius der überdeckenden Kreise. Die Kreismittelpunkte liegen auf einem Großkreis mit den Polen $P$ und $P^{\prime}$ [Abb. 10a].

\section{Die separierenden Großkreise bilden ein Mosaik vom Typ B}

$\boldsymbol{n}=\mathbf{8 , 1 2}, \mathbf{1 6}$ : Es sei $\gamma=\frac{180^{\circ}}{k-1}$, wobei $k=3,4$ oder 5 , je nachdem, ob $n=8,12$ oder 16. Wir betrachten eine vollständig separierbare Überdeckung der Kugel, die aus $n$ kongruenten Kreisen vom Radius $r$ besteht, und bei der die separierenden Kreise ein Mosaik vom Typ B generieren. Es gibt in dem Mosaik zwei Dreiecksflächen, die sich zu einem Kugelzweieck ergänzen, wobei $180^{\circ}>\gamma^{*} \geq \gamma$. Das bedeutet, dass der Flächeninhalt von einem der Dreiecke mindestens $\gamma^{*}$ ist.

Es sei $A B^{\prime} C$ ein solches Dreieck, wobei $\gamma^{*}$ bei $C$ liegt, und der Bogen $C A$ mindestens so groß wie $C B^{\prime}$ ist. Dadurch ist $C A \geq 90^{\circ}$ und $C A+C B^{\prime} \geq 180^{\circ}$. Daraus folgt, dass das Kugeldreieck $A B^{\prime} C$ ein Kugeldreieck $A B C$ enthält, für das $\varangle A C B=\gamma$ und $C B=180^{\circ}-C A \leq C A$ gilt. Das Dreieck $A B^{\prime} C$ (und dadurch auch $A B C$ ) lässt sich durch einen Kreis vom Radius $r$ überdecken, folglich sind alle Seiten von $A B C$ höchstens $2 r$ [Abb. 11a].

Wir suchen den kleinsten überdeckenden Kreis für das Dreieck $A B C$.

a) Liegt der Umkreismittelpunkt außerhalb von $A B C^{8}$, dann ist $\varangle A B C=\beta>90^{\circ}+\frac{\gamma}{2}$.

${ }^{8}$ Der Umkreismittelpunkt liegt genau dann außerhalb des Dreiecks, wenn es einen Winkel gibt, der größer ist als die Summe der beiden anderen. 
Aus dem Kosinussatz bezüglich der Winkel erhalten wir

$$
\cos A C=\frac{\cos \alpha \cdot \cos \gamma+\cos \beta}{\sin \alpha \cdot \sin \gamma}=\frac{\cos \beta(1-\cos \gamma)}{\sin \beta \cdot \sin \gamma}=\operatorname{ctg} \beta \cdot \operatorname{tg} \frac{\gamma}{2}
$$

Nach den Voraussetzungen $180^{\circ}>\beta>90^{\circ}+\frac{\gamma}{2}$ und $\operatorname{ctg} \beta<\operatorname{ctg}\left(90^{\circ}+\frac{\gamma}{2}\right)=-\operatorname{tg} \frac{\gamma}{2}$ folgt eine Abschätzung bezüglich $A C$ zwischen $90^{\circ}$ und $180^{\circ}$ :

$$
\cos A C<-\left(\operatorname{tg} \frac{\gamma}{2}\right)^{2}=\frac{\cos 2 r_{n}}{\cos ^{2} r_{n}}<\cos 2 r_{n}, \text { d.h. } 2 r \geq A C>2 r_{n}
$$

Ein Dreieck mit äußerem Umkreismittelpunkt liefert keinen besseren Wert für den Radius des überdeckenden Kreises als der durch die Konstruktion gegebene.

b) Der Fall des inneren Umkreismittelpunktes: Der Radius des überdeckenden Kreises ist mindestens so groß wie der Umkreisradius. Den Umkreisradius $r^{*}$ vergleichen wir mit $r_{n}$ durch eine elementare Methode ${ }^{9}$.

Ist $C A=C B$, dann $r \geq r^{*}=r_{n}$.

Ist $C A>C B$, dann betrachten wir den Umkreis $K$ von $A B C$. Der Kreismittelpunkt wird durch $O$ bezeichnet. Wir zeichnen durch $C$ zwei Großkreise, die den Winkel $\gamma$ haben, und $C O$ ist die Winkelhalbierende von $\gamma$. Der Kreis $\mathrm{K}$ schneidet die zwei Großkreise in $A_{1}$ und $B_{1}$ ( $B_{1}$ liegt auf dem Kreisbogen $A B$, der den Punkt $C$ nicht enthält; $A_{1}$ liegt auf dem Bogen $A C$, der den Punkt $B$ nicht enthält) [Abb. 11b].

Es wird gezeigt, dass $A B>A_{1} B_{1}$ gilt:

Wir drehen das Kugeldreieck $A_{1} B_{1} C$ um $O$ mittels $\varangle B_{1} O B$ so, dass der Bildpunkt von $B_{1}$ mit $B$ übereinstimmt. Das Bild von $A_{1}$ und von $C$ bezeichnen wir mit $A_{2}$ bzw. $C_{2}$. Es ist bekannt, dass der Peripheriewinkel in jedem von $C_{2}$ unterschiedlichen Punkt des $C_{2}$ enthaltenden Bogens $A_{2} B$ kleiner ist als der Peripheriewinkel $A_{2} C_{2} B$. (Da $C$ ein solcher Punkt ist, gilt dies auch für $C$.) Der Endpunkt $A$ des Bogens $C A$, der mit $C B$ einen Winkel $\gamma$ bildet, liegt deswegen auf dem Bogen $A_{2} B$, der den Punkt $C$ enthält. Wir wissen, dass $A_{1} B_{1}$ und $A_{2} B$ gleich lang sind. So gilt für die (den Punkt $C$ nicht enthaltenden) Bögen $A B>A_{1} B_{1}$ [Abb. 11c].

Es wird gezeigt, dass der Kreis K einen größeren Teil der Fläche des Zweiecks $A_{1} C B_{1}$ abschneidet als des Zweiecks $A C B$ :

Da der von dem Zweieck $A C B_{1}$ abgeschnittene Teil in beiden Bereichen enthalten ist, genügt es, die von den Zweiecken $A_{1} C A$ und $B_{1} C B$ abgeschnittenen Teile zu vergleichen [Abb. 12a].

${ }^{9}$ Ähnlich der Idee von G. Fejes Tóth 1969. 
Um diese zu vergleichen, drehen wir den Kreis $K$ um den Punkt $C$ mittels $\gamma$ so, dass das Bild von $C B$ mit dem Schenkel $C A$ übereinstimmt. (Die Bilder von $K, A, B, A_{1}$ und $B_{1}$ werden mit $K^{\prime}, A^{\prime}, B^{\prime}, A_{1}^{\prime}$ bzw. $B_{1}^{\prime}$ bezeichnet.)

Wegen $C A>C B$ liegt $B^{\prime}$ innerhalb des Bogens $C A$. Aus $\varangle A_{1} C B_{1}=\gamma$ und $C A_{1}=C B_{1}$ folgt, dass $C A_{1}$ die gemeinsame Sehne von $K$ und $K^{\prime}$ ist. Das durch $C A_{1}$ abgeschnittene (kleinere) Segment von $K^{\prime}$ liegt innerhalb des größeren Segments des Kreises ${ }^{10} K$. Der Flächeninhalt des Schnittes von $K$ mit dem Winkelbereich $B C A$ ist kleiner als der Flächeninhalt des Schnittes von $K$ mit dem Winkelbereich $B_{1} C A_{1}$ [Abb. 12b].

Die Dreiecke $A B C$ und $A_{1} B_{1} C$ werden bezüglich des Flächeninhalts verglichen:

Werden die durch $A B$ bzw. $A_{1} B_{1}$ weggeschnittenen Segmente weggelassen, entstehen die Dreiecke $A B C$ bzw. $A_{1} B_{1} C$. Da $A B$ größer ist als $A_{1} B_{1}$ und der Bereich (aus dem das kleinere Segment weggelassen wurde) größer war, gilt nun, dass der Flächeninhalt des gleichschenkligen Dreiecks $A_{1} B_{1} C$ größer ist als der Flächeninhalt von $A B C$ [Abb. 12c].

Es wurde also gezeigt, dass der Kreis vom Radius $r$ ein gleichschenkliges Dreieck $A_{1} B_{1} C$ enthält, dessen Flächeninhalt größer als $\gamma$ ist, folglich hat ein gleichschenkliges Dreieck mit dem Flächeninhalt $\gamma$ einen kleineren Umkreis: $r \geq r^{*}>r_{n}$.

\section{Die separierenden Großkreise bilden ein Mosaik vom Typ C}

Für $\boldsymbol{n}=\mathbf{1 4}$ wird das Mosaik vom Typ C durch 4 Großkreise generiert. Dadurch entstehen (nach dem Eulerschen Polyedersatz) 8 Dreiecke und 6 Vierecke. Die Schnittpunkte der gegenüberliegenden Seiten einer ausgewählten Vierecksfläche bestimmen je ein diametral gegenüberliegendes Punktpaar $\left(P, P^{\prime}\right)$ und $\left(Q, Q^{\prime}\right)$, die auf einem - von den separierenden Großkreisen unterschiedlichen - Großkreis $\Gamma$ liegen ${ }^{11}$. Der Großkreis $\Gamma$ schneidet die Kugel in zwei Halbkugeln und zerlegt die Eckpunkte des Mosaiks in 3 Klassen: 4 Eckpunkte liegen auf $\Gamma, 4$ weitere auf einer offenen Halbkugel, 4 auf der anderen offenen Halbkugel. Daraus folgt, dass jede Vierecksfläche entlang jeder Kante je einer Dreiecksfläche benachbart ist. Aus der Anzahl von Dreiecks- bzw. Vierecksflächen folgt, dass die Kantennachbarn von Dreiecken Vierecke werden müssen. Topologisch handelt es sich um ein Mosaik vom Typ $(4,3,4,3)$.

${ }^{10}$ Das ist eben das Spiegelbild des durch $C A_{1}$ abgeschnittenen kleineren Segments $-C A_{1}$ ist kleiner als die Diagonale von $K, O$ liegt innerhalb von $K$.

${ }^{11}$ Die 4 Schnittpunkte $P, P^{\prime}, Q, Q^{\prime}$ sind gleichzeitig Eckpunkte des Mosaiks. 
$P, P^{\prime}, Q, Q^{\prime}$ zerlegen den Großkreis $\Gamma$ in 4 Bögen, folglich ist einer mindestens $90^{\circ}$ lang. Diese Bögen sind Diagonalen von je einer - zu der ausgewählten Vierecksfläche durch den Eckpunkt anschließenden - Vierecksfläche. Das bedeutet, dass der Radius des überdeckenden Kreises (mindestens in einem Fall) mindestens $45^{\circ}$ sein muss. Im Falle von $45^{\circ}$ entsteht die dünnste vollständig separierbare Überdeckung, die separierenden Großkreise bilden ein halbreguläres Mosaik $(4,3,4,3)$ [Abb. 13].

Damit wurde der folgende Satz bewiesen:

SATz. Für die Radien $r_{n}$ der dünnsten Überdeckungen der Kugel durch $n \leq 16$ vollständig separierbare kongruente Kreise gilt:

$$
\begin{aligned}
& r_{2}=r_{4}=r_{6}=r_{10}=90^{\circ} ; \\
& r_{8}=\operatorname{arcctg} \frac{\sqrt{2}}{2} \approx 54,735^{\circ} ; \\
& r_{12}=\operatorname{arcctg} \frac{\sqrt{3}}{2} \approx 49,106^{\circ} ; \\
& r_{14}=45^{\circ} ; \\
& r_{16}=\operatorname{arcctg} \sqrt{\frac{\sqrt{2}+2}{4}} \approx 47,275^{\circ} .
\end{aligned}
$$

Eine einfache Überlegung zeigt, dass die Lage der Kreise der dünnsten vollständig separierbaren Überdeckung der Kugel durch kongruente Kreise für $n=2,8,12,16$ (bis auf Kongruenz) eindeutig ist, jedoch für $n=4,6,10$ nicht eindeutig ist. Bei der Überdeckung durch 14 Kreise enthält jeder Kreis je eine Fläche des halbregulären Mosaiks $(4,3,4,3)$. Die Lage der Umkreise der Vierecksflächen ist eindeutig. Die überdeckenden Kreise der Dreiecksflächen können ein wenig „schwappen“.

\section{B) Medieneinsatz, Studentenaktivität}

Die Studierenden haben den Text selbständig gelesen. Um die Vermutung zu beweisen bzw. die Beweisschritte nachzuvollziehen, haben sie eigene Skizzen bzw. Berechnungen angefertigt. Beim Standpunktwechsel wurden die Lénárt-Kugel und Cabri-Darstellungen verwendet (Liste der Animationen). 


\section{Zusammenfassung}

Durch die hier skizzierte Fallstudie wurden u.a. die folgenden Aussagen verstärkt:

- Die bewusst eingesetzte Medienkombination kann - durch Analogiebildung - in relativ kurzer Zeit zur Einsicht führen, auch wenn die Studierenden auf dem Gebiet keine genaue Vorstellung haben.

- Die diversen Darstellungen der Sachverhalte (konkret-manipulativ auf der Kugel, bewegliche sphärische Figuren am Bildschirm, statische Abbildungen) und der (mathematisch korrekt formulierte) Text präzisieren einander gegenseitig und erleichtern so ein tieferes Verständnis.

- Das kreative Umgehen mit dem Lehrstoff ist nicht nur beim Entdecken eines neuen Zusammenhanges zu fördern, sondern auch beim verständnisvollen Nachvollzug eines Beweises.

- Die Komplexität der Aufgabe hat sich positiv auf die Bereitschaft der Studierenden in Richtung Nutzung des Medieneinsatzes ausgewirkt.

\section{Literatur}

K. Böröczky, Teljesen szeparálható körelhelyezések a gömbön, Speciálkollégiumi ill. szemináriumi előadás, 1981.

K. Böröczky, The problem of Tammes for $n=11$, Studia Sci. Math. Hungar. 18 (1983), $165-171$.

J. S. Brunner, Some theorems on instruction illustrated with reference to mathematics, in Theories of learning and instruction, The sixty-third yearbook of the National Society for the Study of Education, Part I (306-335), (Hilgard, E.R., ed.), University of Chicago Press., Chicago, 1964.

L. Danzer, Endliche Punktmengen auf der 2-Sphäre mit möglichst großem Minimalabstand, Habilitationsschrift, Univ. Göttingen, 1963.

L. Danzer, Finite point-sets on $S$ with minimum distance as large as possible, Discrete Math. (1975), 60, 3-66.

M. Goldberg, Viruses and a mathematical problem, J. Mol. Biol. 24 (1967), 337-338.

L. Fejes Tóth, Regular figures, Pergamon-Macmillan, New York, 1964.

G. Fejes Tóth, Kreisüberdeckungen der Sphäre, Studia Sci. Math. Hungar. 4 (1969), $225-247$.

G. Fejes Tóth, Totally separable packing and covering with circles, Studia Sci. Math. Hungar. 22 (1987), 65-73.

G. Fejes Tóth \& L. Fejes Tóth, On totally separable domains, Acta Math. Acad. Sci.

Hungar. 24 (1973), 229-232. 
K. J. Fuchs \& É. Vásárhelyi, Geometrie und Algebra - Zwei gleichwertige Partner, in Beiträge zum Mathematikunterricht, Franzbecker, Hildesheim, 1998, 623-626.

L. Hárs, The problem of Tammes for $n=10$, Studia Sci. Math. Hungar. 21 (1986), 439-451.

H.-J. Herber \& É. Vásárhelyi, Das Unterrichtsmodell „Innere Differenzierung einschließlich Analogiebildung“ - Aspekte einer empirisch veranlassten Modellentwicklung, Salzburger Beiträge zur Erziehungswissenschaft Jg. 6, Nr. 2 (2003), 5-19.

G. Kertész, On totally separable packing of equal balls, Acta Math. Hungar. 51 (1988), 363-364.

I. Lénart, K. J. Parisont and É. Vásárhelyi, Erste Schritte mit der Lénárt-Kugel, Abakus Verlag, Salzburg, 2002.

T. W. Melnyk, O. Knop \& W. R. Smith, Extremal arrangements of points and unit charges on a sphere: equilibrium configurations revisited, Canad. J. Chem. 55 (1977), $1745-1761$.

R. M. Robinson, Arrangment of 24 points on a sphere, Math. Ann. 144 (1961), 17-48. K. Schütte \& B. L. van der Waerdden, Auf welcher Kugel haben 5, 6, 7, 8 oder 9. Punkte mit Mindestabstand Eins Platz?, Math. Ann. 123 (1951), 96-124.

K. Schütte, Überdeckung der Kugel mit höchstens acht Kreisen, Math. Ann. 129 (1955), 181-6.

P. M. L. Tammes, On the origin of number and arrangements of the places of exit on the surface of pollen-grains, Recl. Trav. Bot. Neerl. 27 (1930), 1-84.

T. Tarnai, Spherical circle-packings in nature, practice and theory, Structural Topology 9 (1984), 39-58.

B. L. van der Waerden, Pollenkörner, Punktverteilungen und Informationstheorie, Die Naturwissenschaften 48 (1961), 189-192.

É. Vásárhelyi, Optimal placing of convex figures, Dissertation at the Hungarian Academy of Science, Hungarian, 1991.

É. Vásárhelyi, Combination of traditional and computer based tools as a strategy for problem solving, in Meißner, H., Grassmann, M., Müller-Philipp, S. (Hg) Proceedings of the International Conference „Creativity and Mathematics Education“, Münster, 1999, 163-166.

É. Vásárhelyi, Totally separable covering of the sphere by $n \leq 16$ congruent circles - Discover a theorem with a combination of Lénárt sphere and computer animation, Hungarian, 2003a, www.matserv.pmmf.hu/cseri, www.mathdid.inhun.com.

É. Vásárhelyi, Interaktive Arbeitsblätter mit Cabriwin, 2003b, www. mathdid.inhun. com.

M. Wertheimer, Experimentelle Studien über das Sehen von Bewegung, Zeitschrift für Psychologie 61 (1912), 161-265. 
Abbildungen

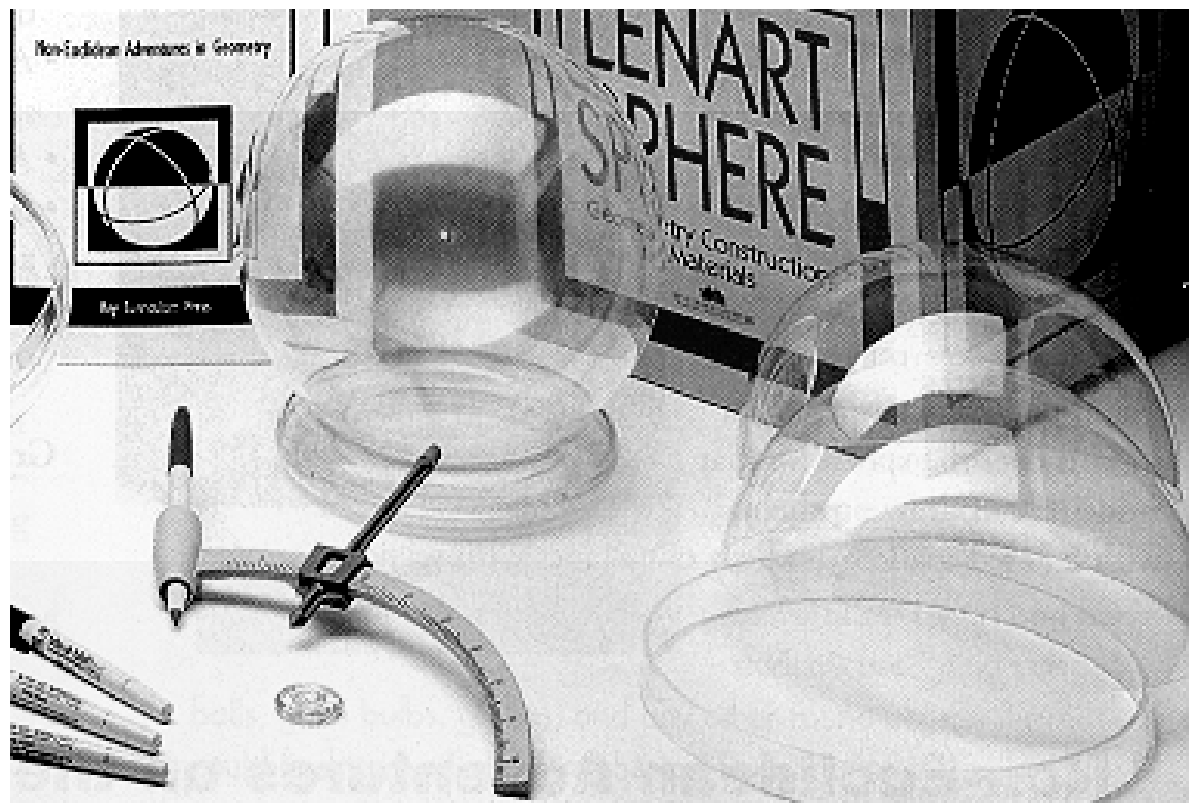

Abbildung 1

Das sphärische Konstruktionswerkzeug, die Lénárt-Kugel.

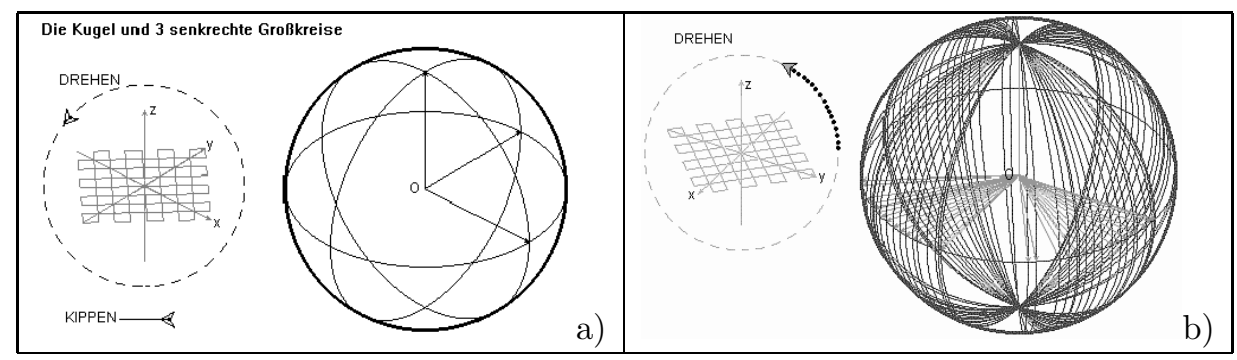

Abbildung 2

Experimentieren mit Cabri-Animation rundumdiekugel1.fig.

a) Eine „günstige“ Projektion wählen.

b) Die „Spuren“ bei einer Drehung. 


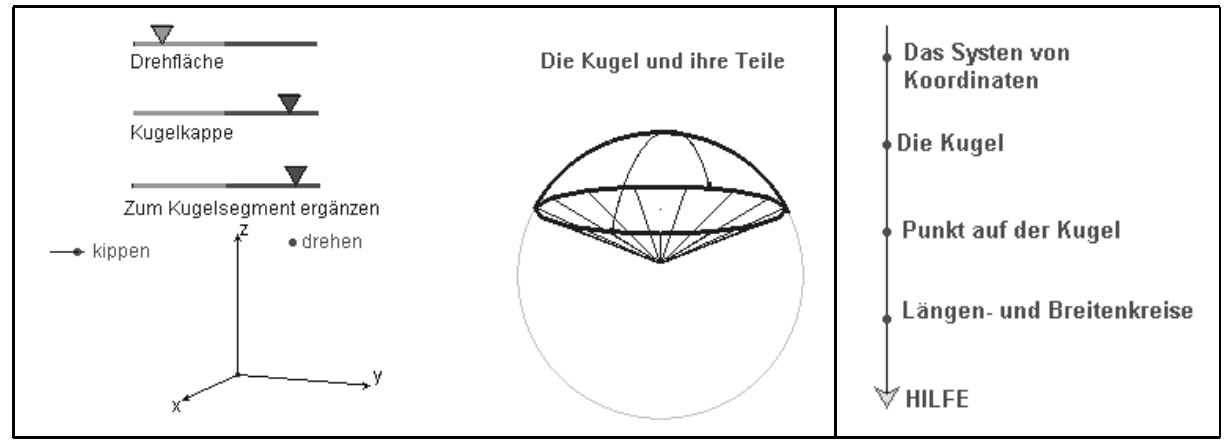

Abbildung 3

Schalter bzw. mehrstufige Hilfeleiste auf den Arbeitsblättern.

(rundumdiekugel0.fig)

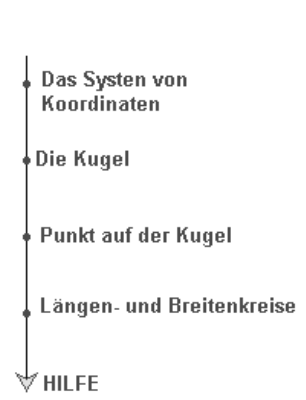

\section{Experimentieren}
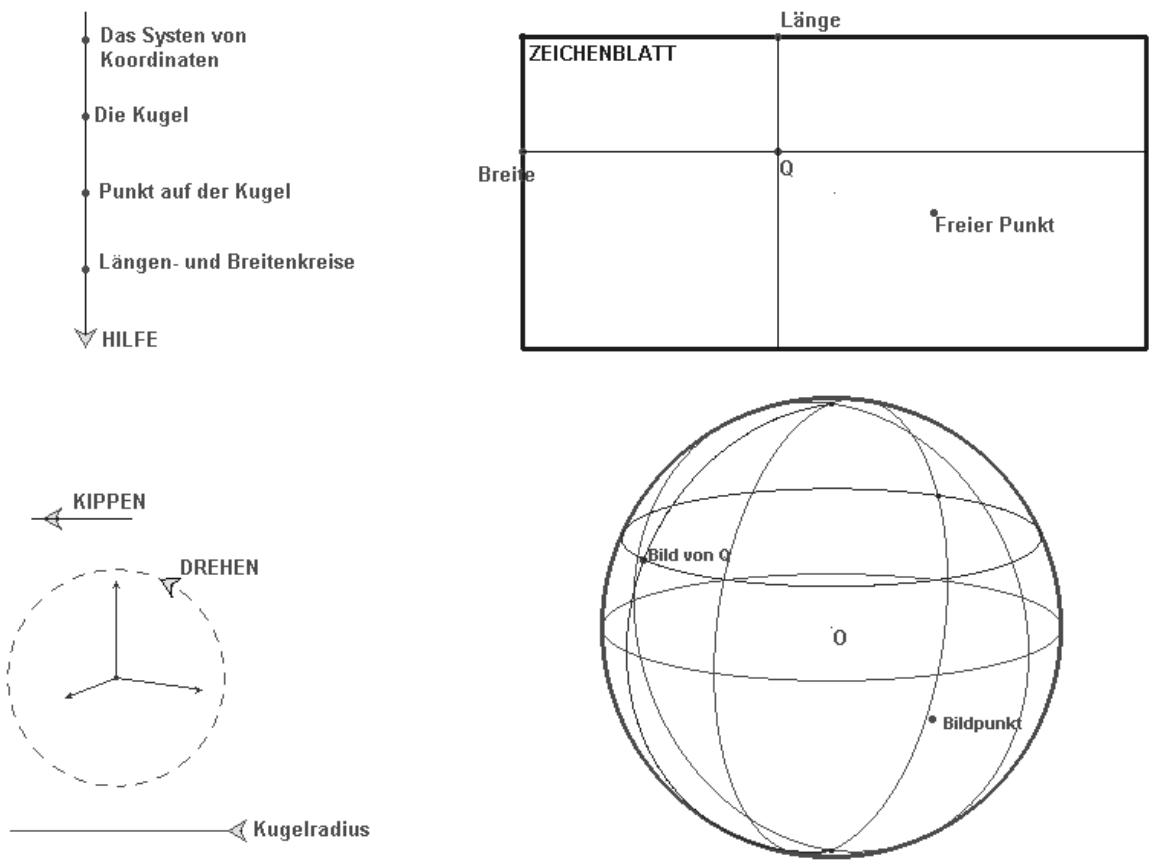

Abbildung 4

Experimentieren mit dem interaktiven Arbeitsblatt. Auf dem Zeichenblatt wer- 
den die Punkte herausgesucht, die auf der Kugel dargestellt werden. „Das Zeichenblatt verbindet" die geographischen Koordinaten und die Koordinaten des Ortsvektors eines Punktes. Der (durch die Parallelprojektion) verzerrte Ortsvektor wird als lineare Kombination der verzerrten Einheitsvektoren berechnet und in einem Koordinatensystem dargestellt, dessen Ursprung das Kugelzentrum ist. (rundumdiekugel.fig)

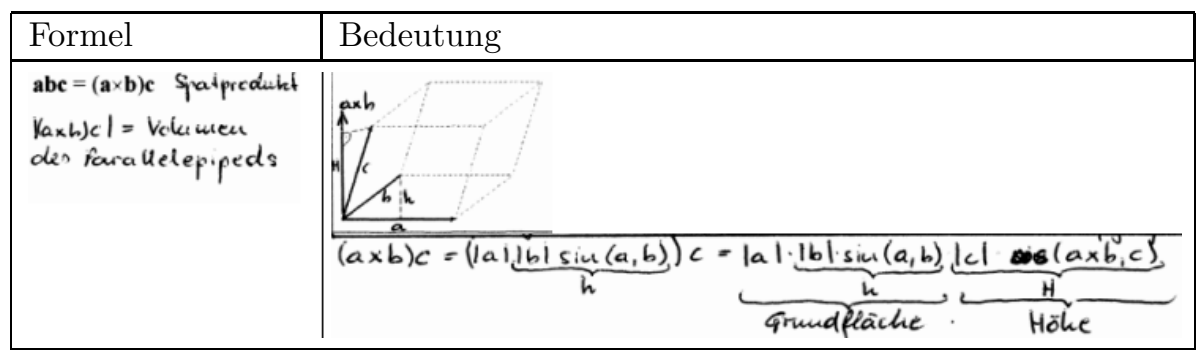

\section{Abbildung 5}

Ausschnitt aus einer Studentenarbeit: Formel und Bedeutung verknüpfen, das Spatprodukt.

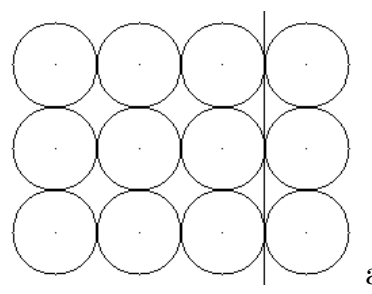

a)

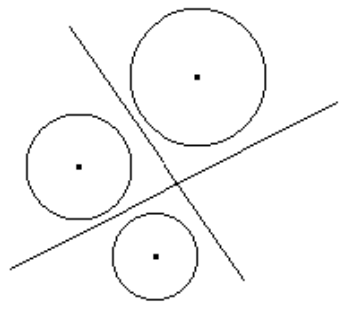

b)

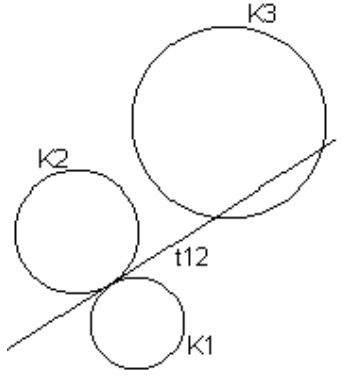

c)

Abbildung 6

Die Figuren a) und b) stellen je ein vollständig separierbares Kreissystem dar, dagegen ist das System in c) nicht vollständig (aber lokal) separierbar, denn die gemeinsame Tangente von $K 1$ und $K 2$ (die einzige Gerade, die $K 1$ und $K 2$ trennt) schneidet den Kreis $K 3$. 


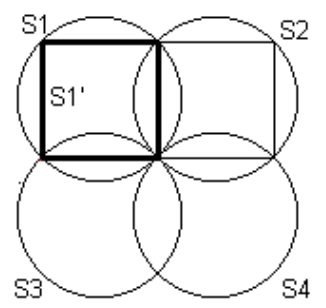

Abbildung 7

Laut Definition 2 ist das Kreissystem $\{S i\}$ vollständig separierbar, denn das Quadratsystem $\left\{S i^{\prime}\right\}$ bildet eine vollständig separierbare Packung.

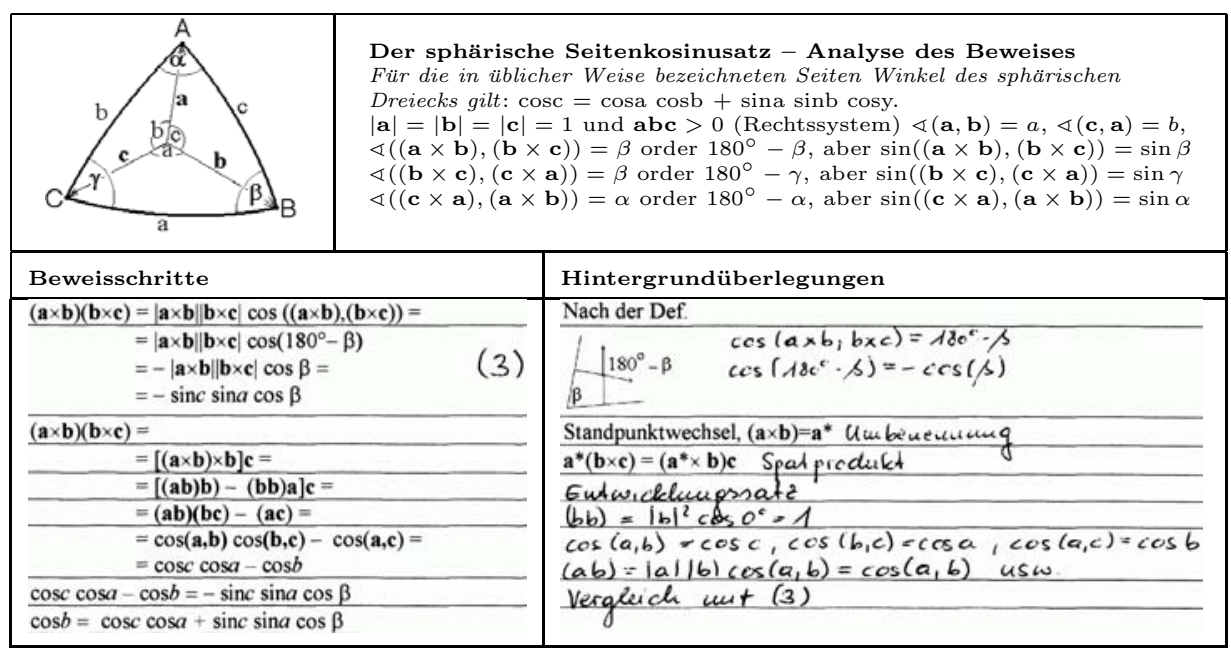

Von welcher Formel hätten wir ausgehen sollen, um den Zusammenhang bezüglich der Seite „c“ zu erhalten? Trick: Die Antwort $(\mathbf{b} \times \mathbf{c})(\mathbf{c} \times \mathbf{a})$ wurde im Rechteck $3 \square$ versteckt, durch Umfärben der Füllfarbe erhalten Sie $(b \times c)(c \times a)$.

\section{Abbildung 8}

Ausschnitt aus einer Studentenarbeit: Der sphärische Seitenkosinussatz - Analyse des Beweises. 


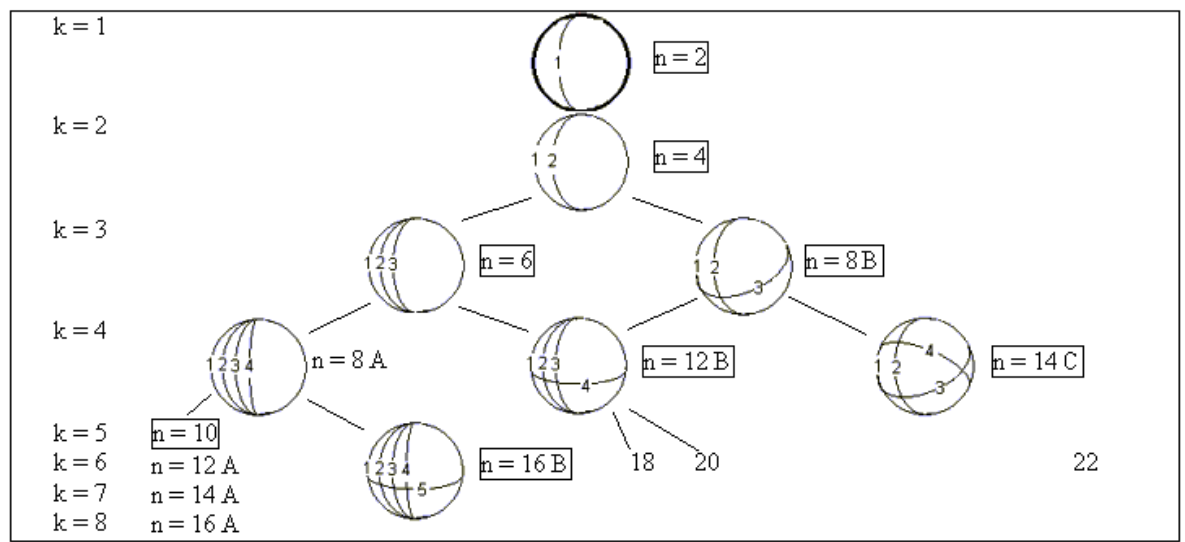

Abbildung 9

Ausschnitt aus einer Studentenarbeit: Die vollständig separierbaren Mosaike werden durch Erhöhen der Anzahl der Großkreise generiert.

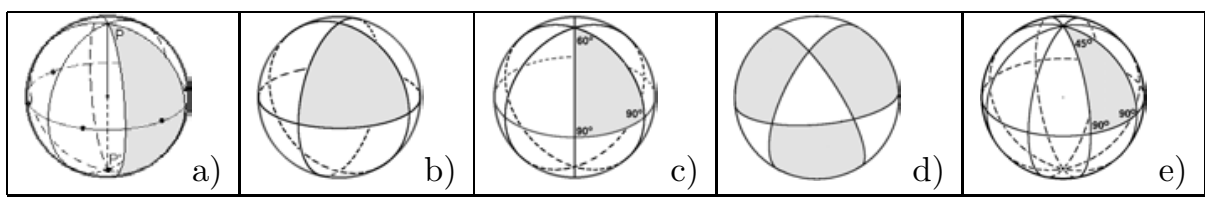

Abbildung 10

Möglichst kleinen Radius suchen durch Konstruktion.

a) Vollständig separierbares Mosaik vom Typ A. Jede Fläche ist ein Kugelzweieck. Die Mittelpunkte der überdeckenden Kreise mit dem Radius $90^{\circ}$ liegen auf einem Großkreis.

b) Das reguläre Mosaik $\{3 ; 4\}$. Die Flächenumkreise bilden eine vollständig separierbare Überdeckung.

c) $r_{12}$ ist der Umkreisradius des gleichschenkligen Dreiecks mit den Winkeln $60^{\circ}, 90^{\circ}, 90^{\circ}$.

d) $r_{14}$ ist der Umkreisradius der Vierecksflächen des halbregulären Mosaiks $(4,3,4,3)$.

e) $r_{16}$ ist der Umkreisradius eines Kugeldreiecks mit den Winkeln $45^{\circ}, 90^{\circ}, 90^{\circ}$. 


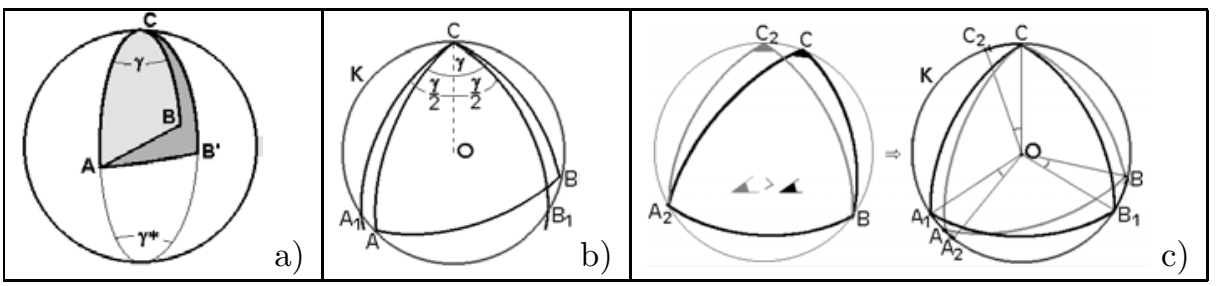

Abbildung 11

Beweisfiguren zu den Fällen $n=8,12,16$.

a) Das Kugelzweieck, von dem $A B^{\prime} C$ abgeschnitten wurde, ist mindestens so groß wie der Mittelwert. In dieses Zweieck wurde ein Zweieck vom Winkel $\gamma$ (Mittelwert) eingeschrieben und halbiert, um ein Dreieck $A B C$ mit dem Flächeninhalt $\gamma$ zu erhalten.

b) $K$ ist der Umkreis des Dreiecks $A B C$, dessen Radius zu untersuchen ist. Das Dreieck $A B C$ wird mit dem gleichschenkligen Dreieck $A_{1} B_{1} C$, dessen Schenkel den Winkel $\gamma$ einschließen, bezüglich des Flächeninhalts verglichen.

c) Das Kugeldreieck $A_{1} B_{1} C$ wird um $O$ mittels $\varangle B_{1} O B$ so gedreht, dass der Bildpunkt von $B_{1}$ mit $B$ übereinstimmt. Das Bild von $A_{1}$ und von $C$ wurde mit $A_{2}$ bzw. $C_{2}$ bezeichnet.

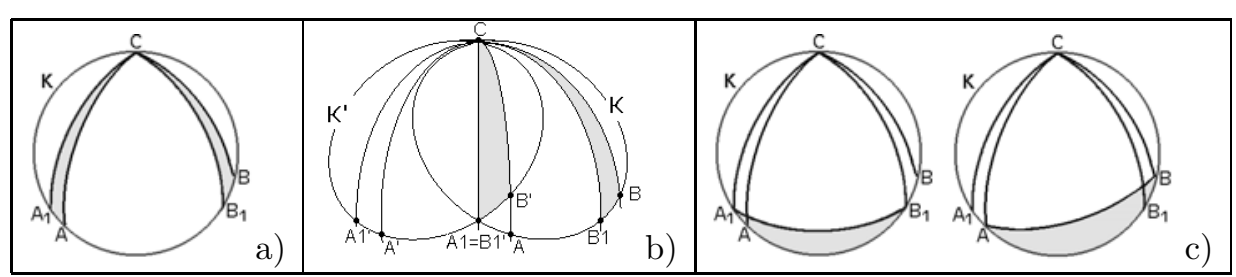

Abbildung 12

a) Es werden die durch $K$ von dem Zweieck $A_{1} C B_{1}$ und von dem Zweieck $A C B$ abgeschnittenen Bereiche bezüglich des Flächeninhalts verglichen.

b) Der Kreis $K$ wird um den Punkt $C$ mittels $\gamma$ so gedreht, dass das Bild des Schenkels $C B$ mit dem Schenkel $C A$ übereinstimmt. Der durch $K$ von $A_{1} C A$ abgeschnittene Bereich enthält das Bild des von $B_{1} C B$ abgeschnittenen Bereiches.

c) Da $A B>A_{1} B_{1}$ gilt, wird ein größeres Segment von $A C B$ weggelassen als von $A_{1} C B_{1}$. 


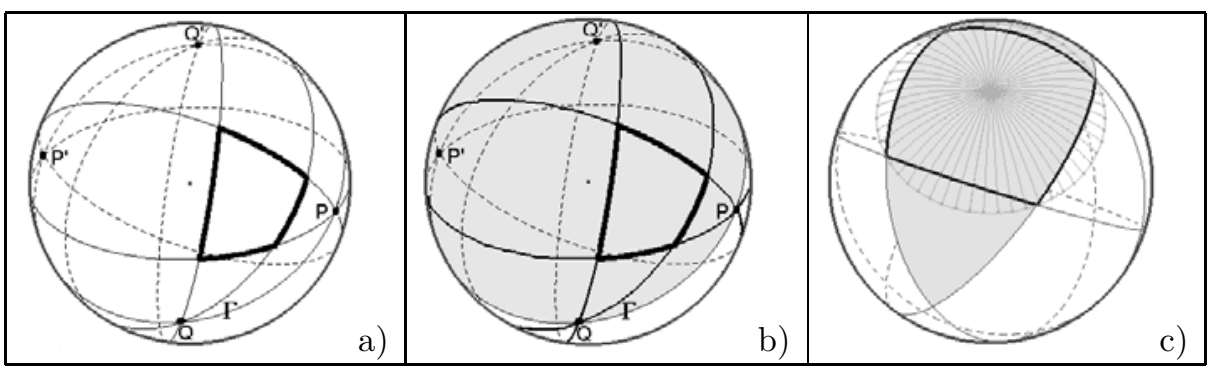

Abbildung 13

Gesucht wird der kleinstmögliche Radius für die vollständig separierbare Überdeckung der Kugel durch 14 kongruente Kreise:

a) Eine Viereckfläche und die topologische Struktur $(4,3,4,3)$ des Mosaiks vom Typ C.

b) Es gibt mindestens einen Bogen auf $\Gamma$ der nicht kürzer als $90^{\circ}$ ist (entweder $P Q$ oder $Q P^{\prime}$, denn sie bilden insgesamt $180^{\circ}$ ).

c) Sind alle Bögen gleich lang, entsteht das halbreguläre Mosaik $(4,3,4,3)$. In der Figur sind eine Viereckfläche (in der Mitte des Kugelzweiecks) und der überdeckende Umkreis des Vierecks zu sehen. 


\section{Liste der Animationen}

3dhilfe.fig: $\quad$ Steuerungselemente der Animationen

rundumdiekugel0.fig: Die Kugel und ihre Teile

rundumdiekugel1.fig: Die Kugel und 3 senkrechte Großkreise

rundumdiekugel2.fig: Hintergrund der Darstellung

rundumdiekugel3.fig: Es werden Punkte auf die Kugel gezeichnet

rundumdiekugel4.fig: Es werden Parallelkreise auf die Kugel gezeichnet

rundumdiekugel5.fig: Es werden Längenkreise auf die Kugel gezeichnet

rundumdiekugel6.fig: Es werden Spiralen auf die Kugel gezeichnet

rundumdiekugel7.fig: Es werden Großkreise (Geraden) auf die Kugel gezeichnet

rundumdiekugel8.fig: Es werden Kugeldreiecke auf die Kugel gezeichnet

rundumdiekugel.fig: Experimentieren mit sphärischen Figuren

ÉVA VÁSÁRHELYI

EÖTVÖS LORÁND UNIVERSITÄT

NATURWISSENSCHAFTLICHE FAKULTÄT

MATHEMATISCH-FACHDIDAKTISCHE GRUPPE

H-1117 BUDAPEST, PÁZMÁNY PÉTER SÉTÁNY 1/C

HUNGARY

BZW

UNIVERSITÄT SALZBURG

INSTITUT FÜR ERZIEHUNGSWISSENSCHAFT

ABTEILUNG FÜR LEHR-/LERNFORSCHUNG

INSTRUKTIONSMEDIEN UND SCHULENTWICKLUNG

A-5020 SALZBURG, AKADEMIESTRASSE 26

AUSTRIA

E-mail: vasar@ludens.elte.hu, Eva.Vasarhelyi@sbg.ac.at

(Received July, 2003) 